\title{
LQG Control Approach for Gaussian MAC with Feedback
}

\author{
Ehsan Ardestanizadeh and Massimo Franceschetti \\ Electrical and Computer Engineering \\ University of California, San Diego \\ La Jolla, CA, 92093-0407, USA \\ E-mail: ehsan@ucsd.edu, massimo@ece.ucsd.edu
}

\begin{abstract}
The communication problem over the $N$-sender additive white Gaussian noise (AWGN) multiple access channel (MAC) with feedback is considered. It is shown that Kramer's code, which is known to be sum rate optimal in the class of generalized linear feedback codes, can be obtained by solving a linear quadratic Gaussian (LQG) control problem. This generalizes the previous result of Elia, which recovers Ozarow's code (a special case of Kramer's code for $N=2$ ) using different approach based on Youla parametrization. Our construction is based on a general correspondence between AWGN-MAC and a linear time invariant (LTI) system controlled over a point-topoint AWGN channel which could be useful in other contexts.
\end{abstract}

\section{INTRODUCTION}

The capacity region of the additive white Gaussian noise (AWGN) multiple access channel (MAC) with feedback is not known in general. In the special case of $N=2$, Ozarow [1] established the capacity region which, unlike the point-to-point case, turns out to be strictly larger than that without feedback. The code proposed by Ozarow is an extension of the Schalkwijk-Kailath code [2] for point-topoint AWGN channels. Later, Kramer [3] generalized these codes for $N>2$. Under equal power constraints for all the senders, Kramer's code achieves the sum capacity provided that the power exceeds a certain threshold depending on the number of senders [3]. On the other hand, for all positive equal power constraints, Kramer's code is known to be optimal within the subclass of generalized linear feedback codes [4].

In this paper, we show that results from control theory can be used to design codes for the AWGN multiple access channel (MAC) with feedback. We consider the linear time-invariant (LTI) system controlled over a point-to-point AWGN channel depicted in Figure 1b. Based on linear controllers for this system, we characterize the corresponding subclass of LTI codes for the AWGN-MAC depicted in Figure 1a.

The set of achievable rates by LTI codes depends only on the system and not the controller. For a given system, we find the optimal controller with minimum average power, using the theory of linear quadratic Gaussian (LQG) control problems [5]. The asymptotic analysis for the rate-power trade-off of the optimal LTI code, which is derived based on the optimal controller, is then presented. For equal power constraints, we show that the asymptotic performance of the optimal LTI code is same as that of Kramer's code [3], which is based on an estimation approach. Note that optimality here refers only to the subclass of LTI codes described above, and proving optimality among the class of all linear codes requires additional arguments [4]. Furthermore, to the best of our knowledge, it is not yet known whether linear codes are in general optimal.

The idea of using control theory to derive codes for feedback communication is not new. In fact, our result generalizes the previous work of [6], which recovers Ozarow's code, a special case of Kramer's code for $N=2$, using controltheoretic tools. Our approach is different from [6] in both the model and the analysis. Our reduction is to a control problem over a single point-to-point channel for any $N>2$ number of senders, and our analysis is based on the theory of LQG control. In contrast, in [6] communication is limited to 2-sender MAC, which is mapped to a control problem also over a 2-sender MAC, and the analysis is based on the technique of Youla parameterization.

The rest of the paper is as follows. In Section II and Section III we present the problem setup and the LTI codes for the AWGN-MAC, respectively. Section IV shows that the optimal LTI code corresponds to Kramer's code. Section V provides the concluding remarks.

\section{Definitions And Problem Setup}

Consider the communication problem between $N$ senders and a receiver over a multiple access channel with additive white Gaussian noise (AWGN-MAC) when channel outputs are noiselessly fed back to all the senders (Figure 1a). Each sender $j \in\{1, \ldots, N\}$ wishes to reliably transmit a message $M_{j} \in \mathcal{M}_{j}$ in the unit interval, i.e., $\mathcal{M}_{j}:=(0,1)$, to the receiver. At each time $i$, the output of the channel is

$$
Y_{i}=\sum_{j=1}^{N} X_{j i}+Z_{i}
$$

where $X_{j i} \in \mathbb{R}$ is the transmitted symbol by sender $j$ at time $i, Y_{i} \in \mathbb{R}$ is the output of the channel, and $\left\{Z_{i}\right\}$ is a discrete-time zero-mean white Gaussian noise process with unit average power, i.e., $\mathrm{E}\left(Z_{i}^{2}\right)=1$ and is independent of $M_{1}, \ldots, M_{N}$. We assume that output symbols are causally 


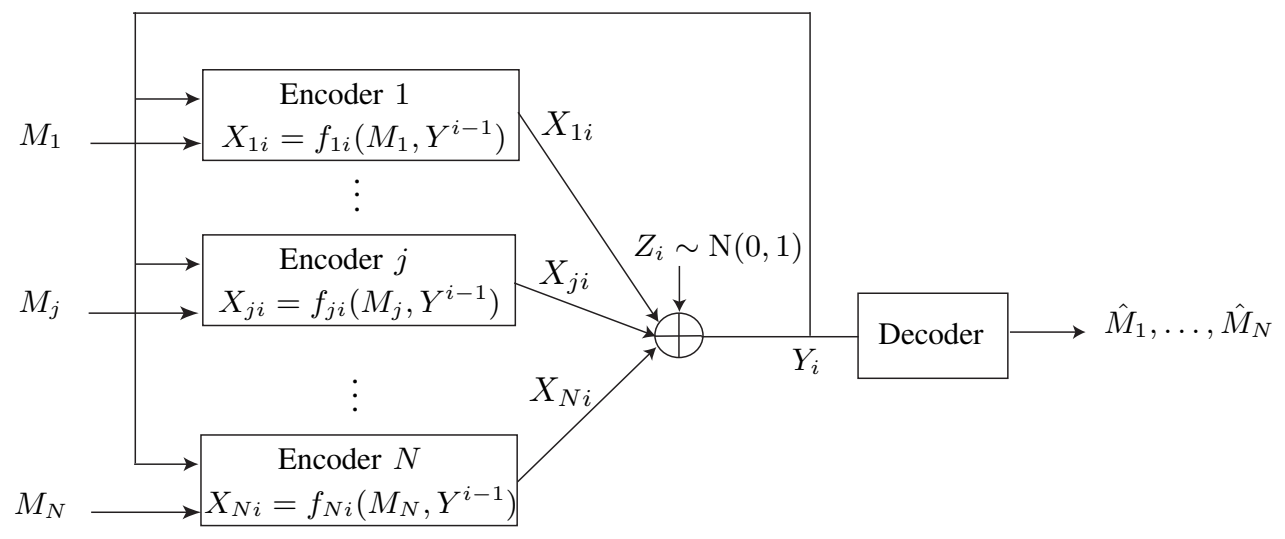

(a) AWGN-MAC with feedback

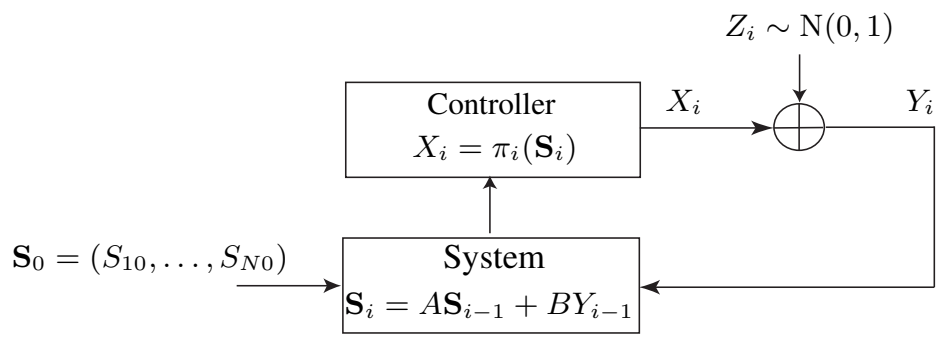

(b) Control over AWGN channel

Fig. 1. A set of $N$ encoders for the feedback communication over AWGN-MAC depicted in (a), can be represented as a dynamical system controlled over AWGN channel depicted in (b).

fed back to the sender and the transmitted symbol $X_{j i}$ for sender $j$ at time $i$ can depend on both the message $M_{j}$ and the previous channel output sequence $Y^{i-1}:=$ $\left\{Y_{1}, Y_{2}, \ldots, Y_{i-1}\right\}$.

Definition 1: We define an $n$-code for the AWGN-MAC as

1) $N$ encoders: each encoder $j, j=1, \ldots, N$ is a set of encoding maps $f_{j i}:\left(\mathcal{M}_{j}, \mathcal{Y}^{i-1}\right) \rightarrow \mathcal{X}_{j}, i=1, \ldots, n$, also known to the receiver, such that

$$
X_{j i}=f_{j i}\left(M_{j}, Y^{i-1}\right)
$$

and

2) a decoder: a decoding map $\phi: \mathcal{Y}^{n} \rightarrow \mathcal{M}_{1} \times \ldots \times$ $\mathcal{M}_{N}$ which determines the estimates of the messages $\hat{M}_{1}, \ldots, \hat{M}_{N}$ given $Y^{n}$

$$
\left(\hat{M}_{1}, \ldots, \hat{M}_{N}\right)=\phi\left(Y^{n}\right) .
$$

We assume that $\mathbf{M}:=\left(M_{1}, \ldots, M_{N}\right) \sim \operatorname{Unif}\left((0,1)^{N}\right)$ and the performance measure is the set of mean square errors

$$
D_{j}^{(n)}:=\mathrm{E}\left(\left(M_{j}-\hat{M}_{j}\left(Y^{n}\right)\right)^{2}\right) \quad j=1, \ldots, N .
$$

Definition 2: The MSE exponents $\left(E_{1}, \ldots, E_{N}\right)$ are called achievable with asymptotic powers $\left(\bar{P}_{1}, \ldots, \bar{P}_{N}\right)$ if there exists a sequence of $n$-codes such that for $j=1, \ldots, N$,

$$
E_{j}=\lim _{n \rightarrow \infty}-\frac{1}{2 n} \log \left(D_{j}^{(n)}\right)
$$

and

$$
\bar{P}_{j}=\lim _{n \rightarrow \infty} \mathrm{E}\left(X_{j n}^{2}\right) .
$$

In the following, we review the definition of the informationtheoretic achievable rates [7], and present the relationship between the achievable MSE exponents and the achievable rates. Consider the $n$-code in Definition 1 with discrete message sets $\mathcal{M}_{j, n}:=\left\{1,2, \ldots, 2^{n R_{j}}\right\}$ whose sizes depend on the block length $n$, and let

$$
P_{e}^{(n)}:=\mathrm{P}\left\{\left(M_{1}, \ldots, M_{N}\right) \neq\left(\hat{M}_{1}, \ldots, \hat{M}_{N}\right)\right\} .
$$

Definition 3: The rate-tuple $\left(R_{1}, \ldots, R_{N}\right)$ is called achievable under block power constraints $\left(P_{1}, \ldots, P_{N}\right)$ if there exists a sequence of $n$-codes with independent messages $\left\{M_{j}\right\}_{j=1}^{N}$ uniformly distributed over $\left\{\mathcal{M}_{j, n}\right\}_{j=1}^{N}$ such that

$$
\sum_{i=1}^{n} \mathrm{E}\left(X_{j i}^{2}\right) \leq n P_{j}, \quad j \in\{1, \ldots, N\}
$$

and $P_{e}^{(n)} \rightarrow 0$ as $n \rightarrow \infty$.

We refer to $R=\sum_{j=1}^{N} R_{j}$ as the sum rate of a given code.

Recall that the MSE exponent $E_{j}$ is defined based on a message $M_{j} \in(0,1)$ which does not depend on the block length $n$. The following lemma provides the connection between the achievable rates and the MSE exponents.

Lemma 1: Let MSE exponents $\left(E_{1}, \ldots, E_{N}\right)$ with asymptotic powers $\left(\bar{P}_{1}, \ldots, \bar{P}_{N}\right)$ be achievable and

$$
R_{j}<E_{j}, \bar{P}_{j}<P_{j}
$$


for $j=1, \ldots, N$. Then rate-tuple $\left(R_{1}, \ldots, R_{N}\right)$ is also achievable and the block power constraints $\left(P_{1}, \ldots, P_{N}\right)$ are asymptotically satisfied.

Proof: See Appendix A.

According to Lemma 1, showing that MSE exponent $E_{j}>$ $R_{j}$ is achievable for the uniform message $M_{j} \in(0,1)$ is sufficient to show that the achievability of rate $R_{j}$, defined based on discrete message sets $\mathcal{M}_{j, n}:=\left\{1,2, \ldots, 2^{n R_{j}}\right\}$.

\section{LTI CODES FOR AWGN-MAC}

In this section we show that LTI codes can be designed for the AWGN-MAC with feedback, based on the LTI systems controlled over a point-to-point AWGN channel depicted in Figure 1b. Let the state $\mathbf{S}_{i} \in \mathbb{C}^{N}$ be a complex vector of length $N$, and the system dynamics be

$$
\mathbf{S}_{i}=A \mathbf{S}_{i-1}+B Y_{i-1} \quad i=1, \ldots, n
$$

where

$$
A=\left(\begin{array}{ccccc}
\beta_{1} \omega_{1} & 0 & 0 & \ldots & 0 \\
0 & \beta_{2} \omega_{2} & 0 & \ldots & 0 \\
\vdots & \vdots & \vdots & \ddots & \vdots \\
0 & 0 & 0 & \ldots & \beta_{N} \omega_{N}
\end{array}\right), B=\left(\begin{array}{c}
1 \\
1 \\
\vdots \\
1
\end{array}\right)
$$

such that $\beta_{j}>1$ are real and $\left|\omega_{j}\right|=1$ are distinct point on the unit circle of the complex plane. As it is clear from the system dynamics given in (6), the dependence of the state on the channel outputs is causal .

The controller is assumed to control each mode $j$ separately and transmit a combined control signal (scalar) $X_{i}$ as follows. It observes the current state $\mathbf{S}_{i}$, picks a vector

$$
\mathbf{X}_{i}:=\left(X_{1 i}, \ldots, X_{N i}\right)
$$

where

$$
X_{j i}=\pi_{j i}\left(\mathbf{S}_{i}(j)\right), \quad j=1, \ldots, N
$$

can depend only on $\mathbf{S}_{i}(j)$, and then transmits the control signal as

$$
X_{i}=\sum_{j=1}^{N} X_{j i}=\sum_{j=1}^{N} \pi_{j i}\left(\mathbf{S}_{i}(j)\right) .
$$

For the purpose of analysis we assume complex transmissions over a complex channel,

$$
Y_{i}=X_{i}+Z_{i}, \quad Z_{i} \sim \mathcal{C N}(0,1) \text { i.i.d. }
$$

where $X_{i}, Y_{i} \in \mathbb{C}$ are input and output of the channel, respectively, and $\left\{Z_{i}\right\}$ is a discrete-time zero-mean white complex Gaussian noise process with identity covariance. Note that a transmission over a complex channel can be viewed as two transmissions over a real channel and the achievable rates per each complex dimension are achievable over a real channel.

We assume the complex messages $M_{j} \in \mathbb{C}, j=1, \ldots, N$, in the AWGN-MAC are uniform over $(0,1) \times(0,1)$ and we set the initial state $\mathbf{S}_{0} \in \mathbb{C}^{N}$ of the system as

$$
\mathbf{S}_{0}=\mathbf{M}=\left(M_{1}, \ldots, M_{N}\right) .
$$

Given the system (6) and the controller (10), we derive the $n$-code for the AWGN-MAC as follows.

1) Encoding mappings: Encoder $j$ recursively forms

$$
\mathbf{S}_{i}(j)=\beta_{j} \omega_{j} \mathbf{S}_{i-1}(j)+Y_{i-1}, i=1, \ldots, n
$$

and at time $i$ transmits $X_{j i}=\pi_{j i}\left(\mathbf{S}_{i}(j)\right)$.

2) Decoding: The decoder forms the estimate vector $\hat{\mathbf{M}}_{i}$, $i=1, \ldots, n$, recursively as follows,

$$
\hat{\mathbf{M}}_{i}=\hat{\mathbf{M}}_{i-1}-A^{-i} B Y_{i-1}, \quad \hat{\mathbf{M}}_{0}=0, Y_{0}=0
$$

and picks $\hat{\mathbf{M}}_{n}(j)$ as the estimate of the message $M_{j}$ at time $n$.

Note that to design codes for MAC, it is necessary that $A$ be diagonal as in (7) and the control signal be of the form (10) since the encoders in the MAC are decentralized and do not have access to each other's messages. In the code described above, the dynamics of the $j$-th mode of the system $\mathbf{S}_{i}(j)$ represents the information, based on which the encoder $j$ picks the transmitted signal $X_{j i}$ at time $i$. We say that the controller stabilizes the system if

$$
\limsup _{n \rightarrow \infty} \mathrm{E}\left(\left|\mathbf{S}_{n}(j)\right|^{2}\right)<\infty, \quad j=1, \ldots, N .
$$

Lemma 2: If the controller of the form (10) stabilizes the linear system in (6), the corresponding sequence of $n$-codes for the AWGN-MAC with feedback achieves MSE exponents $E_{j}=\log \left(\beta_{j}\right), j=1, \ldots, N$.

Proof: See Appendix B.

Remark 1: Note that the set $\left\{\beta_{j}\right\}$ depends only on the system $^{1}$ and not the controller. Therefore, based on different controllers which stabilize the same system we can design different codes which achieve the same set of MSE exponents.

Let $K_{\mathbf{X}_{i}}:=\operatorname{Cov}\left(\mathbf{X}_{i}\right)$ be the covariance of $\mathbf{X}_{i}$ given in (8), and let the asymptotic covariance $\bar{K}_{\mathbf{X}}$ be $\bar{K}_{\mathbf{X}}:=$ $\lim _{n \rightarrow \infty} \bar{K}_{\mathbf{X}_{n}}$. Then, the asymptotic powers are given by $\bar{P}_{j}:=\left(\bar{K}_{\mathbf{X}}\right)_{j j}$. Consider the stationary linear control of the form

$$
X_{i}=-C \mathbf{S}_{i}, \quad C=\left[c_{1} c_{2} \ldots c_{N}\right]
$$

The following theorem provides the asymptotic powers for these linear controllers.

Theorem 1: Consider the stationary linear controller of the form (14) for system given in (6). If $A-B C$ is stable, i.e., the eigenvalues of $A-B C$ are inside the unit circle, the corresponding code for the AWGN-MAC designed based on this linear control problem achieves the rates

$$
R_{j}<E_{j}=\log \left(\beta_{j}\right), \quad j=1, \ldots, N
$$

with asymptotic powers

$$
\bar{P}_{j}=c_{j}^{2} \bar{K}_{j j}, \quad j=1, \ldots, N
$$

where $\bar{K}$ is the unique solution of the following discrete algebraic Lyapunov equation (DALE)

$$
\bar{K}=(A-B C) \bar{K}(A-B C)^{\prime}+Q, \quad Q=B B^{\prime} .
$$

\footnotetext{
${ }^{1}$ For the rest of the paper, system refer to the system given in (6).
} 
Proof: First note that by Lemma 2, MSE exponents $E_{j}=\log \left(\beta_{j}\right), j=1, \ldots, N$, are achievable for any controller that stabilizes system (6). Moreover, by similar argument as in Lemma 1, one can see that, in each complex dimension, any rate $R_{j}<E_{j}$ is achievable for senders $j=1, \ldots, N$.

To find the asymptotic powers, note that given a stationary linear controller of the form (14), the closed loop system given (6) can be written as

$$
\mathbf{S}_{i}=(A-B C) \mathbf{S}_{i-1}+B Z_{i-1}
$$

Consider the code corresponding to the linear control (14), where the encoder $j$ transmits

$$
X_{j i}=c_{j} \mathbf{S}_{i}(j)
$$

at time $i$. Let $K_{i}:=\operatorname{Cov}\left(\mathbf{S}_{i}\right)$. Then, the asymptotic power $\bar{P}_{j}$ is

$$
\bar{P}_{j}=\lim _{n \rightarrow \infty} \frac{1}{n} \sum_{i=1}^{n} \mathrm{E}\left(X_{j i}^{2}\right)=\lim _{n \rightarrow \infty} \frac{1}{n} \sum_{i=1}^{n}\left|c_{j}\right|^{2}\left(K_{i}\right)_{j j}
$$

From the closed loop dynamic given in (17) we have the following Lyapunov recursion,

$$
K_{n}=(A-B C) K_{n-1}(A-B C)^{\prime}+Q, \quad Q=B B^{\prime}
$$

If $A-B C$ is stable, we know [5] that

$$
\lim _{n \rightarrow \infty} K_{n}=\bar{K} \succ 0
$$

where $\bar{K}$ is the unique postive-definite solution of the corresponding discrete algebraic Lyapunov equation (DALE),

$$
\bar{K}=(A-B C) \bar{K}(A-B C)^{\prime}+Q .
$$

Therefore, by the Cesaro mean theorem and (19) the asymptotic power in (18) becomes $\bar{P}_{j}=\left|c_{j}\right|^{2} \bar{K}_{j j}$.

\section{LQG PRoblem ANd KRAMER's CODE}

As shown in Lemma 2, the set of achievable MSE exponents, or equivalently rates, depend only on the system. For a given system, we find the optimal controller of the form (10), which stabilizes the system, and also minimizes the asymptotic power

$$
\bar{P}:=\lim _{n \rightarrow \infty} \frac{1}{n} \sum_{i=1}^{n} \mathrm{E}\left(\left|X_{i}\right|^{2}\right) .
$$

Note that by (10), the asymptotic power of the controller can be written as $\bar{P}=\sum_{j, k=1}^{N}\left(\bar{K}_{\mathbf{X}}\right)_{j k}$. Hence, $\bar{P}$ represents the asymptotic combined power of all the transmitters in the corresponding LTI code, which also captures the correlation between the transmitted signals.

Lemma 3: The optimal controller of the form (10) which minimizes the cost (20) is stationary and linear, i.e.,

$$
X_{i}=-C \mathbf{S}_{i}
$$

where

$$
C=\left(B^{\prime} G B+1\right)^{-1} B^{\prime} G A
$$

and $G$ is the unique positive-definite solution to the following discrete algebraic Riccati equation (DARE)

$$
G=A^{\prime} G A-A^{\prime} G B\left(B^{\prime} G B+1\right)^{-1} B^{\prime} G A .
$$

Proof: Note that the optimal power $\bar{P}^{*}$ for the controllers of the form (10) can be lower bounded as

$$
\bar{P}^{*} \geq \bar{P}_{\min }
$$

where $\bar{P}_{\text {min }}$ is the optimal cost considering a more general stabilizing controller of the form

$$
X_{i}=\pi_{i}\left(\mathbf{S}_{i}\right)
$$

which picks the scalar action $X_{i}$ based on the complete state $\mathbf{S}_{i}$ and is not necessarily separated for different modes as in (10). Considering the general control (23) we have a linear Gaussian quadratic control problem. From the theory of LQG [5] we know the optimal control is linear, i.e.,

$$
X_{i}=-C_{i} \mathbf{S}_{i}, \quad C_{i}=\left[c_{1 i} c_{2 i} \ldots c_{N i}\right] .
$$

and the stationary linear control $C=\lim _{i \rightarrow \infty} C_{i}$, which minimizes the asymptotic cost (20), is given by $C$ in (21). Note that $G=0$, which is a solution to (22), is not acceptable since the corresponding controller $C=0$ does not stabilize the system in (7), and we are only interested in the stabilizing controllers (see Lemma 2). In fact, since the eigenvalues of $A$ are all outside of the unit circle, we know [8, Lemma 2.4] that the stabilizing solution to (22) is unique and positivedefinite.

Since the controller in (24) is of the form (10) we can conclude

$$
\bar{P}^{*}=\bar{P}_{\min }
$$

and the optimal controller of the form (10) is same as the optimal controller of the LQG problem.

Consider the symmetric system

$$
\begin{aligned}
\omega_{j} & =e^{\frac{2 \pi i}{N}(j-1)} \\
\beta_{j} & =\beta \quad j=1, \ldots, N .
\end{aligned}
$$

and the optimal LTI code for the AWGN-MAC based on the optimal control given in Lemma 3. The following theorem shows that under equal power constraints, this optimal LTI code corresponds to Kramer's code, which is known to be sum rate optimal within the class of generalized feedback codes [4].

Theorem 2: Let $R(P)$ be the sum rate achieved by Kramer's code with equal power $P$. There exists $\beta>1$ such that the optimal LTI code based on the system (25) achieves the sum rate $R(P)$ and satisfies the equal power constraint $P$ asymptotically.

Proof: By Theorem 1, the optimal LTI code based on the symmetric system (25) and the optimal controller in (21) achieves the symmetric MSE exponent $E$, i.e, $E_{j}=E=\log (\beta), j=1, \ldots, N$ with asymptotic powers $\bar{P}_{j}=c_{j}^{2} \bar{K}_{j j}, j=1, \ldots, N$ where $\bar{K}$ is given in (16). Moreover, by Lemma 1 , rates $R_{j}<\log \left(\beta_{j}\right), j=1, \ldots, N$ are achievable and as a result sum rate

$$
R<N \log (\beta)
$$


is achievable.

On the other hand, Let $R(P)$ denote the sum rate achievable by Kramer's code under symmetric per-symbol power $P$. From [3] we know

$$
R(P)<\frac{1}{2} \log (1+N P \phi(P))
$$

where $\phi(P) \in \mathbb{R}$ is the unique solution, in interval $[1, N]$, for

$$
(1+N P \phi)^{N-1}=(1+P \phi(N-\phi))^{N} .
$$

Therefore, by picking

$$
R(P)<N \log (\beta)<\log (1+N P \phi(P))
$$

the LTI code achieves sum rate equal to $R(P)$.

It remains to show that the asymptotic powers in the optimal LTI code satisfy

$$
\bar{P}_{j}<P, \quad j=1, \ldots, N .
$$

Towards this end, first we find the asymptotic powers $\bar{P}_{j}$. Note that the DARE given in (22) can be equivalently written as the following discrete algebraic Lyapunov equation (DALE)

$$
G=(A-B C)^{\prime} G(A-B C)+C^{\prime} C .
$$

From (16) and (31) we have

$$
G_{j j}=c_{j}^{2} \bar{K}_{j j}
$$

and from (15) and (32) we conclude that the asymptotic power of the encoder $j$ in the optimal LTI code is determined by the matrix $G$ as

$$
\bar{P}_{j}=G_{j j} .
$$

The following lemma characterizes the matrix $G$ for the symmetric choice of $A$ given in (25).

Lemma 4: Let $\beta_{j}=\beta$ and $\omega_{j}=e^{2 \pi i \frac{(j-1)}{N}}$. Then the unique positive-definite solution $G \succ 0$ of (22) is circulant with real eigenvalues satisfying $\lambda_{i}=\frac{1}{\beta^{2}} \lambda_{i-1}$ for $i=2, \ldots, N$. The largest eigenvalue $\lambda_{1}$ satisfies

$$
\begin{gathered}
1+N \lambda_{1}=\beta^{2 N} \\
\left(1+\lambda_{1}\left(N-\frac{\lambda_{1}}{G_{j j}}\right)\right)=\beta^{2(N-1)} .
\end{gathered}
$$

Proof: See Appendix C.

From (29) and (34) we have

$$
N \log (\beta)=\frac{1}{2} \log \left(1+N \lambda_{1}\right)<\frac{1}{2} \log (1+N P \phi(P))
$$

and hence

$$
\lambda_{1}<P \phi(P) .
$$

Moreover, from (34) and (35) we have

$$
\left(1+N \lambda_{1}\right)^{N-1}=\left(1+\lambda_{1}\left(N-\frac{\lambda_{1}}{G_{j j}}\right)\right)^{N} .
$$

Comparing (37) with condition (28), we can see that

$$
\lambda_{1}=G_{j j} \phi\left(G_{j j}\right) .
$$

Combining with (36) we have $G_{j j} \phi\left(G_{j j}\right)<P \phi(P)$. Since $\phi(P)$ is monotone in $P$ we have $G_{j j}<P$ and (30) follows from (33).

\section{CONCLUSION}

This paper considered the design of codes for the AWGNMAC with feedback based on the linear time invariant (LTI) system controlled over a point-to-point Gaussian channel. The LQG problem is considered in which the objective is to minimize the power of the controller. The codes for the AWGN-MAC with feedback, which corresponds to the optimal solution of this LQG problem, were analyzed and shown to have the same asymptotic performance as that of Kramer's codes, under equal power constraints.

Considering LTI systems, we are limited to linear codes for the communication problems. To include all class of codes, the correspondence between communication and control needs to go beyond linear control. In that case, techniques from nonlinear control could potentially be helpful to obtain fundamental limits in the communication problems. We plan to investigate this general connection in our future work.

\section{APPENDIX A \\ PROOF OF LEMMA 1}

Consider a sequence of $n$-codes for $M_{j} \in(0,1), j=$ $1, \ldots, N$, such that

$$
E_{j}=\lim _{n \rightarrow \infty}-\frac{1}{2 n} \log \left(D_{j}^{(n)}\right), \quad j=1, \ldots, N .
$$

We show that rate $R_{j}<E_{j}$ is achievable. Consider

$$
\begin{aligned}
\mathrm{P}\left(\left|M_{j}-\hat{M}_{j}\right|>\frac{1}{2} \cdot 2^{-n R_{j}}\right) & \leq 4 \cdot 2^{2 n R_{j}} \cdot D_{j}^{(n)} \\
& \leq 4 \cdot 2^{2 n R_{j}} \cdot 2^{-2 n\left(E_{j}-\epsilon_{n}\right)} \\
& =4 \cdot 2^{-2 n\left(E_{j}-R_{j}-\epsilon_{n}\right)}
\end{aligned}
$$

for some $\epsilon_{n}$ where $\epsilon_{n} \rightarrow 0$ as $n \rightarrow \infty$, where (39) follows from Chebyshev inequality and (40) follows from (38). If $R_{j}<E_{j}$, from (41) we have

$$
\mathrm{P}\left(\left|M_{j}-\hat{M}_{j}\right|>\frac{1}{2} \cdot 2^{-n R_{j}}\right) \rightarrow 0
$$

as $n \rightarrow \infty$ and the decoder can pick intervals $\Delta_{j}^{(n)}$ such that $\Delta_{j}^{(n)} \mid=2^{-n R_{j}}$ and

$$
\mathrm{P}\left(M_{j} \notin \Delta_{j}^{(n)}\right) \rightarrow 0 \text { as } n \rightarrow \infty .
$$

By Lemma II.3 in [9], if condition (42) is satisfied then for all $n$, we can map the message set $\mathcal{M}_{n}=\left\{1, \ldots, 2^{n R_{j}}\right\}$ into a set of message points $\tilde{\mathcal{M}}_{n} \in(0,1)$ in the unit interval such that the minimum distance between the two message points is $2^{-n R}$. Therefore, using the decoder with property (42), $P_{e}^{(n)} \rightarrow 0$ as $n \rightarrow \infty$ and rate $R$ is achievable. Moreover, if $\bar{P}_{j}=\lim _{n \rightarrow \infty} \mathrm{E}\left(X_{j n}^{2}\right), j=1, \ldots, N$ and $\bar{P}_{j}<P_{j}$, it is not hard to see that the block power constraint (4) is satisfied for sufficiently large $n$. 


\section{APPENDIX B \\ PROOF OF LEMMA 2}

We can rewrite the system dynamics given in (6) as $\mathbf{S}_{i}=$ $A^{i} \mathbf{S}_{0}+\hat{\mathbf{S}}_{i}$ where

$$
\hat{\mathbf{S}}_{i}=A \hat{\mathbf{S}}_{i-1}+B Y_{i-1}, \quad \hat{\mathbf{S}}_{0}=0, Y_{0}=0
$$

Comparing (43) and (13), we can see that $\hat{\mathbf{M}}_{i}=-A^{-i} \hat{\mathbf{S}}_{i}$. Therefore, $\hat{\mathbf{S}}_{i}=\mathbf{S}_{i}-A^{i} \mathbf{S}_{0}=\mathbf{S}_{i}-A^{i} \mathbf{M}$, and $\hat{\mathbf{M}}_{i}=$ $-A^{-i} \hat{\mathbf{S}}_{i}=\mathbf{M}-A^{-i} \mathbf{S}_{i}$. Hence, the error vector $\mathbf{e}_{n}$, at time $n$, is given by $\mathbf{e}_{n}:=\mathbf{M}-\hat{\mathbf{M}}_{n}=A^{-n} \mathbf{S}_{n}$. Then we have

$$
D_{j}^{(n)}=\mathrm{E}\left(\left|\mathbf{e}_{n}(j)\right|^{2}\right)=\beta_{j}^{-2 n} K_{n}(j, j) .
$$

where $K_{n}:=\operatorname{Cov}\left(\mathbf{S}_{n}\right)$ is the covariance matrix of $\mathbf{S}_{n}$. By the assumption of stability $\lim \sup _{n \rightarrow \infty} K_{n}(j, j)<\infty$ and $E_{j}=\log \left(\beta_{j}\right)$ follows from (44) and definition of $E_{j}$.

\section{APPENDIX C \\ PROOF OF LEMMA 4}

Let $A$ and $B$ be of the form (7) with symmetric parameters as in (25). Then we show that the DARE in (22) has a unique circulant positive-definite solution $\tilde{G} \succ 0$ with real eigenvalues satisfying $\lambda_{i}=\frac{1}{\beta^{2}} \lambda_{i-1}$ for $i=2, \ldots, N$, and the largest eigenvalue $\lambda_{1}$ satisfies

$$
\begin{gathered}
1+N \lambda_{1}=\beta^{2 N} \\
\left(1+\lambda_{1}\left(N-\frac{\lambda_{1}}{\tilde{G}_{j j}}\right)\right)=\beta^{2(N-1)} .
\end{gathered}
$$

Note that any circulant matrix can be written as $Q \Lambda Q^{\prime}$, where $Q$ is the $N$ point DFT matrix with

$$
Q_{j k}=\frac{1}{\sqrt{N}} e^{-2 \pi i(j-1)(k-1) / N},
$$

and $\Lambda=\operatorname{diag}\left(\left[\lambda_{1}, \ldots, \lambda_{N}\right]\right)$ is the matrix with eigenvalues on its diagonal. Plugging $Q \Lambda Q^{\prime}$ into (22) and rearranging we get

$$
\begin{aligned}
& \Lambda=\left(Q^{\prime} A Q\right) \Lambda\left(Q^{\prime} A Q\right)^{\prime}-\left(\left(Q^{\prime} A Q\right) \Lambda\left(Q^{\prime} B\right)\right) \\
& \left(1+B^{\prime} Q \Lambda Q^{\prime} B\right)^{-1}\left(\left(Q^{\prime} A Q\right) \Lambda\left(Q^{\prime} B\right)\right)^{\prime} .
\end{aligned}
$$

For $A$ with symmetric parameters as in (25), we have

$$
Q^{\prime} A Q=\beta\left(\begin{array}{ccccc}
0 & 1 & 0 & \ldots & 0 \\
0 & 0 & 1 & \ldots & 0 \\
\vdots & \vdots & \ddots & \ddots & \vdots \\
0 & 0 & \ldots & 0 & 1 \\
1 & 0 & \ldots & 0 & 0
\end{array}\right), Q^{\prime} B=\left(\begin{array}{c}
\sqrt{N} \\
0 \\
\vdots \\
0
\end{array}\right) \text {. }
$$

Hence,

$$
\begin{aligned}
\left(Q^{\prime} A Q\right) \Lambda\left(Q^{\prime} A Q\right)^{\prime} & =\beta^{2}\left(\begin{array}{cccc}
\lambda_{2} & 0 & \ldots & 0 \\
0 & \lambda_{3} & \ldots & 0 \\
\vdots & \vdots & \ddots & \vdots \\
0 & 0 & \ldots & \lambda_{1}
\end{array}\right) \\
\left(Q^{\prime} A Q\right) \Lambda\left(Q^{\prime} B\right) & =\left(\begin{array}{c}
0 \\
0 \\
\vdots \\
\beta \lambda_{1} \sqrt{N}
\end{array}\right)
\end{aligned}
$$

and the Riccati equation (22) becomes $N$ diagonal equations in terms of the eigenvalues of the circular matrix. The first $N-1$ equations are

$$
\lambda_{j}=\beta^{2} \lambda_{j+1}, \quad j=1, \ldots, N-1
$$

and the $N$-th equation is

$$
\lambda_{N}=\beta^{2} \lambda_{1}-\frac{\beta^{2} \lambda_{1}^{2} N}{1+N \lambda_{1}} .
$$

From (47) we see that $\lambda_{1}$ is the largest eigenvalue and $\lambda_{N}=$ $\beta^{-2(N-1)} \lambda_{1}$. Combining with (48) we get

$$
\left(1+N \lambda_{1}\right)=\beta^{2 N} \text {. }
$$

Hence, $\lambda_{1}$ is real and so are $\lambda_{j}, j=2, \ldots, N$. Note that from the form of $Q$ in (46), $\lambda_{1}=\sigma_{1}$ where

$$
\sigma_{j}:=\sum_{k=1}^{N} \tilde{G}_{j k} .
$$

Moreover, since $\tilde{G}$ is circulant we know that $\sigma_{j}=\sigma_{1}$ for all $j$, and $\left(1+B^{\prime} \tilde{G} B\right)=1+N \lambda_{1}$. Hence, the diagonal equations of the original Riccati equation

$$
\tilde{G}_{j j}=\beta^{2} \tilde{G}_{j j}-\beta^{2} \frac{\sigma_{j}^{2}}{\left(1+B^{\prime} \tilde{G} B\right)}
$$

are equivalent to

$$
\beta^{2}=\frac{1+N \lambda_{1}}{1+\lambda_{1}\left(N-\frac{\lambda_{1}}{\tilde{G}_{j j}}\right)}
$$

and by (49) we have

$$
\left(1+\lambda_{1}\left(N-\frac{\lambda_{1}}{\tilde{G}_{j j}}\right)\right)=\beta^{2(N-1)} .
$$

Combining (47), (49), and (51) completes the proof.

\section{REFERENCES}

[1] L. H. Ozarow, "The capacity of the white Gaussian multiple-access channel with feedback," IEEE Trans. Inf. Theory, vol. 30, pp. 623-629, 1984.

[2] J. P. M. Schalkwijk and T. Kailath, "A coding scheme for additive noise channels with feedback-part I: No bandwidth constraint," IEEE Trans. Inf. Theory, vol. 12, pp. 172-182, 1966.

[3] G. Kramer, "Feedback strategies for white Gaussian interference networks," IEEE Trans. Inf. Theory, vol. 48, pp. 1423-1438, 2002.

[4] E. Ardestanizadeh, M. A. Wigger, T. Javivi, and Y. H. Kim, "Linear sum capacity for Gaussian multiple access channel with feddback," 2010, arXiv:1002.1781.

[5] T. Kailath, A. H. Sayed, and B. Hassibi, Linear Estimation. 2nd ed. New York: Wiley, 2006.

[6] N. Elia, "When Bode meets Shannon: Control oriented feedback communication schemes," IEEE Trans. Automated Control, vol. 47, pp. 1477-1488, 2004.

[7] T. M. Cover and J. A. Thomas, Elements of Information Theory. 2nd ed. New York: Wiley, 2006.

[8] Y.-H. Kim, "Feedback capacity of stationary Gaussian channels," IEEE Trans. Inf. Theory, vol. 56, pp. 57-85, 2010.

[9] O. Shayevitz and M. Feder, "Optimal feedback communication via posterior matching," preprint, http://arxiv.org/abs/0909.4828. 